\title{
Tumor Lysis Syndrome as a Consequence of Chronic Lymphocytic Leukemia Treatment With Fludarabine and Rituximab
}

\author{
Firas Yazigia, , Anuradha Kollurua, Ayad M Al-katib
}

\begin{abstract}
A seventy-nine year old Caucasian male with a diagnosis of chronic lymphocytic leukemia presented with acute tumor lysis syndrome after treatment with two courses of fludarabine and one course of rituxan. In this article we report this rare complication, which has been reported only few times in the literature, and discuss the ways to prevent it from happening.
\end{abstract}

Keywords: Tumor lysis syndrome; Chronic lymphocytic leukemia; Treatment; Fludarabine; Rituximab

\section{Introduction}

Chronic lymphocytic leukemia (CLL) is one of the chronic lymphoproliferative disorders (lymphoid neoplasms). It is characterized by a progressive accumulation of functionally incompetent lymphocytes, which are monoclonal in origin. CLL is the most common lymphoproliferative disorder and the most common leukemia in the western world and occurs mainly in older age population. It usually progresses slowly over an extended period of time and is monitored by following the lymphocytosis, peripheral adenopathy and splenomegaly that ensue. Treatment is rendered to those patients who become symptomatic or when they become anemic or thrombocytopenic.

Acute tumor lysis syndrome is the term applied to a group of metabolic complications that usually occur after the treatment of neoplastic disorders. The findings that may be seen include hyperphosphatemia, hypocalcemia (due to

Manuscript accepted for publication June 9, 2011

${ }^{\mathrm{a}}$ Department of Internal Medicine, St. John Hospital and Medical
Center, Michigan, USA
${ }^{\mathrm{b}}$ Corresponding author: Firas Yazigi, Email: firas.yazigi@stjohn.org

doi:10.4021/jmc239w precipitation of calcium phosphate), hyperuricemia, hyperkalemia, and acute renal failure [1].

Tumor lysis syndrome after fludarabine therapy is extremely uncommon, but may be associated with significant morbidity and mortality.

\section{Case Report}

A 79 year-old Caucasian male with past medical history significant for chronic lymphocytic leukemia (CLL), presented with change in mental status and confusion.

The patient was diagnosed with CLL in 2004, but did not require treatment at that time. He was free of symptoms until a year later when he became fatigued and short of breath and was found to have increased white cell count of 23,000/ $\mathrm{mm} 3$. Chemotherapy was initiated at that point, and the patient received 6 cycles of chlorambucil/prednisone between February 2005 and February 2006.

The patient responded well to treatment with no complications and was being observed thereafter with no intervention until he became dyspneic again in September 2006 and was found to have large right malignant pleural effusion consistent with CLL. At that point the decision was made to start treatment with rituxan and fludarabine.

The patient received $375 \mathrm{mg} / \mathrm{m}^{2}(750 \mathrm{mg})$ of rituxan and $25 \mathrm{mg} / \mathrm{m}^{2}(50 \mathrm{mg})$ of fludarabine as a first dose, and on the next day he received a second dose of fludarabine. Later that day he became very weak, lethargic and difficult to arouse so he was admitted to the hospital for further evaluation.

The patient was found to be in acute renal failure with a creatinine of $2.9 \mathrm{mg} / \mathrm{dl}$. His uric acid was as high as 21.8 $\mathrm{mg} / \mathrm{dl}$, and his potassium was $7.1 \mathrm{mmol} / \mathrm{l}$, phosphorus of $11.7 \mathrm{mg} / \mathrm{dl}$, calcium of $8 \mathrm{mg} / \mathrm{dl}$, WBC of $180,000 / \mathrm{mm} 3$ with lymphocytes of $85 \%$, and platelets of $83,000 / \mathrm{mm} 3$. These findings were consistent with a diagnosis of tumor lysis syndrome. Chest $\mathrm{x}$-ray showed large right pleural effusion, and CT of the head was negative.

During his hospital course the patient was intubated and transferred to the MICU secondary to respiratory failure and progressive confusion. He was started on aggressive IV hydration, and Elitek (Rasburicase) $3 \mathrm{mg}$ as a single dose. 
Chest tube was also placed to drain the large pleural effusion. Continuous venovenous hemodialysis (CVVHD) was initiated by nephrology service to enhance recovery process.

The patient's condition improved remarkably in the following days and upon his full recovery he was transferred to pulmonary rehabilitation facility.

\section{Discussion}

The tumors most frequently associated with the tumor lysis syndrome are the high grade, very aggressive lymphomas, such as Burkitt lymphoma, and the leukemias, particularly acute lymphoblastic leukemia (ALL) and less often acute myeloid leukemia [1-3].

Since the original description of predominantly acute uric acid nephropathy following the treatment of lymphoma and leukemia [1], the presentation of this syndrome has changed in two ways: 1) The introduction of allopurinol, and to a lesser extent uricase, before the onset of antitumor therapy has reduced, but not eliminated, the incidence of acute uric acid nephropathy. Acute renal failure following therapy is now more frequently associated with severe hyperphosphatemia. 2) Spontaneous acute renal failure prior to therapy is now more commonly recognized and is usually due to acute uric acid nephropathy. These patients have increased uric acid production and hyperuricosuria due to the high rate of tumor cell turnover.

The tumor lysis syndrome should be suspected in patients with a large tumor burden who develop acute renal failure in the presence of marked hyperuricemia (greater than $15 \mathrm{mg} / \mathrm{dL}$ or $893 \mu \mathrm{mol} / \mathrm{L}$ ) and/or hyperphosphatemia (greater than $8 \mathrm{mg} / \mathrm{dL}$ or $2.56 \mathrm{mmol} / \mathrm{L}$ ). These levels are in contrast to most other forms of acute renal failure in which the plasma uric acid concentration is less than $12 \mathrm{mg} / \mathrm{dL}$ ( 714 $\mathrm{mol} / \mathrm{L}$ ) and the plasma phosphate level is less than $6 \mathrm{mg} / \mathrm{dL}$ (1.92 $\mathrm{mmol} / \mathrm{L})$ [4].

While only 6 cases of TLS associated with fludarabine therapy have been reported since 1989 (up to 2011) [5, 6], cautious monitoring of patients treated with fludarabine should be undertaken, even after completion of the chemotherapy course. The same applies to the patients who are about to be treated with rituxan (especially after the first dose), since there have been two cases reported of Rituxaninduced TLS in CLL patients in the literature.

Patients about to receive chemotherapy or radiation for a malignancy with rapid cell turnover should be pretreated, if possible, for at least two days with allopurinol plus fluid loading (with saline and possibly mannitol) to maintain a high urine output (greater than $2.5 \mathrm{~L} /$ day) [7]. Reversible forms of renal insufficiency (e.g., volume contraction, hypercalcemia, urinary tract obstruction) should be corrected prior to therapy.

For those unable to receive oral medications, intrave- nous allopurinol was approved in 1996 by the FDA under orphan drug status, and has been commercially available for this indication since 1999 (Aloprim, Nabi, Boca Raton, FL) $[8,9]$. A review of its use in over 1100 patients found that this preparation was both safe and effective.

TLS after fludarabine therapy is extremely uncommon, but may be associated with significant morbidity and mortality. Hence it is important to anticipate such a complication before initiating therapy with fludarabine and rituxan, and apply all available preventive measures to reduce its incidence.

\section{Conflict of Interest}

None

\section{References}

1. Kjellstrand CM, Cambell DC, 2nd, von Hartitzsch B, Buselmeier TJ. Hyperuricemic acute renal failure. Arch Intern Med. 1974;133(3):349-359.

2. Tsokos GC, Balow JE, Spiegel RJ, Magrath IT. Renal and metabolic complications of undifferentiated and lymphoblastic lymphomas. Medicine (Baltimore). 1981;60(3):218-229.

3. Hande KR, Garrow GC. Acute tumor lysis syndrome in patients with high-grade non-Hodgkin's lymphoma. Am J Med. 1993;94(2):133-139.

4. Stapleton FB, Strother DR, Roy S, 3rd, Wyatt RJ, McKay CP, Murphy SB. Acute renal failure at onset of therapy for advanced stage Burkitt lymphoma and B cell acute lymphoblastic lymphoma. Pediatrics. 1988;82(6):863869.

5. Frame JN, Dahut WL, Crowley S. Fludarabine and acute tumor lysis in chronic lymphocytic leukemia. N Engl J Med. 1992;327(19):1396-1397.

6. Calvo-Villas JM, Urcuyo BM, Umpierrez AM, Sicilia F. Acute tumor lysis syndrome during oral fludarabine treatment for chronic lymphocytic leukemia. Role of treatment with rasburicase. Onkologie. 2008;31(4):197199.

7. Razis E, Arlin ZA, Ahmed T, Feldman EJ, Puccio C, Cook $\mathrm{P}$, Chun $\mathrm{HG}$, et al. Incidence and treatment of tumor lysis syndrome in patients with acute leukemia. Acta Haematol. 1994;91(4):171-174.

8. Smalley RV, Guaspari A, Haase-Statz S, Anderson SA, Cederberg D, Hohneker JA. Allopurinol: intravenous use for prevention and treatment of hyperuricemia. J Clin Oncol. 2000;18(8):1758-1763.

9. Feusner J, Farber MS. Role of intravenous allopurinol in the management of acute tumor lysis syndrome. Semin Oncol. 2001;28(2 Suppl 5):13-18. 